\title{
Sound medicine: an introduction to cacophonology
}

$\mathrm{T}$

he Canada Health Act proscribes extra billing for "medically necessary" services; however, the meaning of this crucial phrase remains obscure and its interpretation is clouded by the societal trend to medicalize all aspects of our lives. But ambiguity smiles on creativity, and cash-strapped physicians may find opportunities to provide services before they are elevated to medical necessity, and for which extra billing may be allowed.

To illustrate, consider the newly opened Centre for Auditory and Melodic Recovery (CAMR). This is a very private institution dedicated to the advancement of cacophonology (the study of cochlear vibratory dysphoria), an experimental branch of medicine that has yet to be recognized by provincial medical billing schemes. This venture was inspired by the epidemic of casualties of industrial Muz ${ }^{\star} k$. Such canned music comprises processed sound products from which the vital germ has been extracted, leaving only a husk of impotent rhythm and sterile assonance. Overexposure to these pathogenic strains may arise from careless lingering in department store elevators or hotel washrooms, leading to a restless foot syndrome (involuntary tapping) and pervasive emotional insensibility - the early signs of what is now recognized as deficiency of vitamin $M$, also known as musica appassionata or alimento di amore.

A severe form of cacophonopathology, metallicus gravis, has also been identified among many of the misguided souls who followed the siren of cultura popularis. Victims of metallicus gravis attend mass gatherings to participate in this form of auditory abuse, which employs sound to numb rather than to enhance awareness. In its later stages, patients demonstrate involuntary movement disorders, such as caput metallicus (headbanging), florid hemiballismus (air guitar syndrome) and precipitous projectile collapse (crowd surfing). Post-mortem findings include scarred cerebral gyri, which assume the texture of hard pebbles or rocks, diagnostic of dementia zeppelophilia plumbea. A related condition is black $\mathrm{s}^{\star}$ bbath excephalobaty (BSE), which features Ozzyform band degeneration and afflicts those who dismember flying ro$\circ$ dents with their teeth.

A range of milder disorders involving psycho-acoustic distress have been identified. These include a variety of forms of cochlear burnout, ennui and stress-related audile malaise. Automated directory rage, for example, is precipitated by attempts to contact government offices followed by endless enumerated redirections. Repeated episodes may result in crippling phonic fatigue. Labyrinthine intussusception may result from attempts to understand the benefits of proposed health care reforms. Finally, classical musicians who "slum it" by jamming with exponents of popular music may incur the spiritual torments of highbrow-myalgia.

\section{What treatment options are available?}

The Healthy Listening Program is a primary prevention initiative that educates patients to choose a diversity of musical forms, including polyphonic, solo instrumental, choral, atonal and operatic, according to the principles of the sound pyramid. This program is available through schools and through the outpatient aurally transmitted disease clinic.

The centre also has a series of orpheoceutical treatment protocols. For vitamin M deficiency, the centre is proud to offer the only licensed Mellowtone Maintenance Program in Canada. Patients are managed on an outpatient basis with ambulatory tympano-immersion therapy using an intra-aural device and a portable $\mathrm{MP}_{3}$ pump containing graduated content. Patients start with the vacuous strains of country and western, pass through McCartney and Mancini, plateau with Bacharach and step it up again with Sullivan and Lehar until real musical emotional depth can 
be safely experienced. The heart of the musical spirit is thus gently resuscitated.

For the casualties of metallicus gravis, initial signature keylation therapy for concurrent heavy metal poisoning is combined with an outpatient $\mathrm{CD}$-exchange program. Sometimes a preliminary course of selected percussive passages is helpful to ease the transition to a more sonorous music-scape. Hans Sachs' cobbling punctuation of Bechmesser's song, or the resounding chorus of anvils that greets the descent of Loge and Wotan into Nibelheim, may serve this purpose. Then, a combination euphonotherapy protocol should entail a vigorous initial assault with high-octane symphonic agents such as works by Rossini, Schubert or Beethoven. Three cycles of DRDN (Der Ring des Nibelungen) are then administered over a 2-week period. If side effects appear, such as perceived dysrhythmia or tempo deficit disorders, Satie's keyboard repertoire may be introduced as a rescue modality. Adjuvant maintenance therapy may take the form of the Mozart Only Program (MOP), featuring Mozart's later string quintets.

For those refractory to this approach, typically musical derelicts burdened with RAP (a reckless auditory past), a fresh start is recommended with the Intensive Neuronal Reformat Program. This procedure begins with total aural ablation - a prelude of total silence in a darkened room for 24 hours. At the end of this time, while teetering on the edge of sensorydeprived insanity, the patient's straining eardrum picks up a barely audible sound, which becomes louder in infinitesimal stages over the ensuing hours. This consists of selections from the veritable motherlode of Bach's musical genius: the 224 cantatas, the distillation of Iooo years of humanity's quest to find meaning and beauty in the day-to-day struggle for survival.
Finally, stereopsychic surgery is available, for which instruments are required. This procedure can only be selfadministered. Ideally such patients are identified at a young age and undergo lifelong preparation, including selections from the 555 keyboard sonatas of Domenico Scarlatti. The operation begins with the subject seated, instrument poised in golden silence. A sparkling sound explodes into consciousness from the void, followed by the further crystalline notes, clustered chords, agile arpeggios and powerful pauses - the perfect marriage of rhythm and polyphony. An inviting portal appears in the space-time continuum and, awestruck, the patient wanders through lofty hallways in an ancient Iberian palace, its whispering corridors still echoing faintly with the touch of the royal tutor in a distant room. Eventually the roller coaster ends, the strings are still, silence is reasserted and the operation is complete.

The judicial system already recognizes the work of the Centre for Auditory and Melodic Recovery in the struggle against auditory malfeasance. Perpetrators of backstreet stereopsychic operations and musical delinquents whose technical performance repeatedly abuses a perfectly good score may be subject to involuntary commitment to the centre.

The emergence of cacophonology as an important medical and socio-legal field of interest highlights the myopic scope of traditional medicine, as well as the untapped opportunities for income enhancement through the provision of such medically unnecessary, but socially essential, services as those offered at the centre.

\author{
Brian Gosset MD \\ General practitioner \\ Toronto, Ont. \\ Currently on musical parole
}

